Revue scientifique francophone en Communication

organisationnelle

$18 \mid 2000$

Non-verbal, communication, organisation

\title{
Montrer ou dire : la communication graphique dans la course à l'identification
}

Philippe Quinton

\section{OpenEdition}

\section{Journals}

Édition électronique

URL : https://journals.openedition.org/communicationorganisation/2465

DOI : 10.4000/communicationorganisation.2465

ISSN : 1775-3546

\section{Éditeur}

Presses universitaires de Bordeaux

\section{Édition imprimée}

Date de publication : 1 novembre 2000

ISSN : 1168-5549

Référence électronique

Philippe Quinton, « Montrer ou dire : la communication graphique dans la course à l'identification », Communication et organisation [En ligne], 18 | 2000, mis en ligne le 27 mars 2012, consulté le 05 août 2021. URL : http://journals.openedition.org/communicationorganisation/2465 ; DOI : https://doi.org/ 10.4000/communicationorganisation.2465

Ce document a été généré automatiquement le 5 août 2021.

(C) Presses universitaires de Bordeaux 


\title{
Montrer ou dire : la communication graphique dans la course à l'identification
}

\author{
Philippe Quinton
}

1 Les signes non-verbaux jouent un grand rôle dans les organisations, et plus spécialement dans les stratégies d'identification qui exigent souvent de se montrer avant de pouvoir dire, de convaincre ou de se «placer » visuellement avant d'être en mesure d'argumenter verbalement. Le «dire» se veut de plus en plus visuel mais en même temps le visuel ne s'est toujours pas affranchi des interprétations verbales qu'on lui impose, ce qui paraît paradoxal pour une société qui se veut de «l'image ».

2 L'exploration du non-verbal sera limitée ici aux «paroles graphiques » d'identification, en écrit ou en image, sur papier ou sur écran, perçues en premier dans la communication des organisations et essentielles à la construction de leur « image ».

\section{Problématiques d'inscription : le texte-image}

3 On comprendra ainsi par non-verbal ce qui est écrit d'une part et qui ne dépend pas du verbe d'autre part, car les signes non-verbaux sont toujours matérialisés dans ou sur un support (même un geste) selon un dispositif approprié ${ }^{1}$; ils impliquent une forme visuelle, une écriture, qu'elle soit texte et/ou image, iconique ou symbolique. Ecrire c'est concevoir et dessiner, même si l'acte d'inscription se limite souvent à une saisie de données au clavier. Cette inscription est partie prenante d'un contrat de communication comme le montre l'énonciation typographique qui "parle" indépendamment du sens littéral d'un texte. Par exemple, la nuance typographique du nouveau logotype de France Telecom, devenu france telecom, pèse son poids symbolique en tant que manifestation non-verbale d'un changement statutaire et territorial...

4 Les hommes semblent ne pas pouvoir se passer du verbal dans l'interprétation des faits visuels. Ainsi les commanditaires d'un logotype exigent la production d'un discours d'accompagnement (qui vaut interprétation officielle) pour en assurer la 
compréhension; et ailleurs, dans les écrans informatiques, les textes d'aide s'accrochent aux icônes pour les expliquer... Comme l'a montré le Groupe $\mathrm{Mu}^{2}$ à propos de "l'impérialisme linguistique ", tout système de communication ou de signification ne se ramène pourtant pas à ce que l'on peut en dire, ce qui est néanmoins la pratique courante en matière d'analyse d'image ${ }^{3}$.

\section{Verbal vs visuel : dire et/ou montrer}

5 À partir de la distinction fondamentale entre processus verbal et processus visuel, on peut mettre l'axe verbal vs visuel face à son contraire non-visuel vs non-verbal. La structure logique ainsi constituée met en valeur deux modes de communication bien distincts pour les organisations (voir fig. 1) :

- ce qui se voit et ne se dit pas ${ }^{4}($ le non-verbal) : on montre sans dire ;

7 - ce qui se dit et ne se voit pas, (le verbal) : on dit sans montrer, c'est le non-visuel.

8 Ces interactions entre verbal et non-verbal résument les potentiels et les paradoxes constitutifs des messages en texte-image (voir fig. 2), ceux qui soutiennent les stratégies discursives des organisations et satisfont à leurs problématiques d'identification plus ou moins contrôlées ${ }^{5}$. Sur un même support, les actes de parole graphique ont donc une face visuelle et une face verbale. Ce qui ne peut se dire peut se montrer, sachant toutefois que montrer c'est aussi dire (même si l'image ne dit pas tout), et cela concerne évidemment l'énonciation ${ }^{6}$. L'approche sémio-pragmatique des discours visuels se trouve prolongée aujourd'hui dans l'ébauche d'une « sémiotique cognitive $»^{7}$ avec les problématiques de la "pensée visuelle » et de la "pensée figurative ", qui envisagent d'autres manières de concevoir et d'exprimer n'impliquant pas le recours systématique à la langue dans la compréhension des faits graphiques, ce qui n'empêche pas le visuel d'être soumis au filtrage culturel, comme l'a montré Edward Hall'

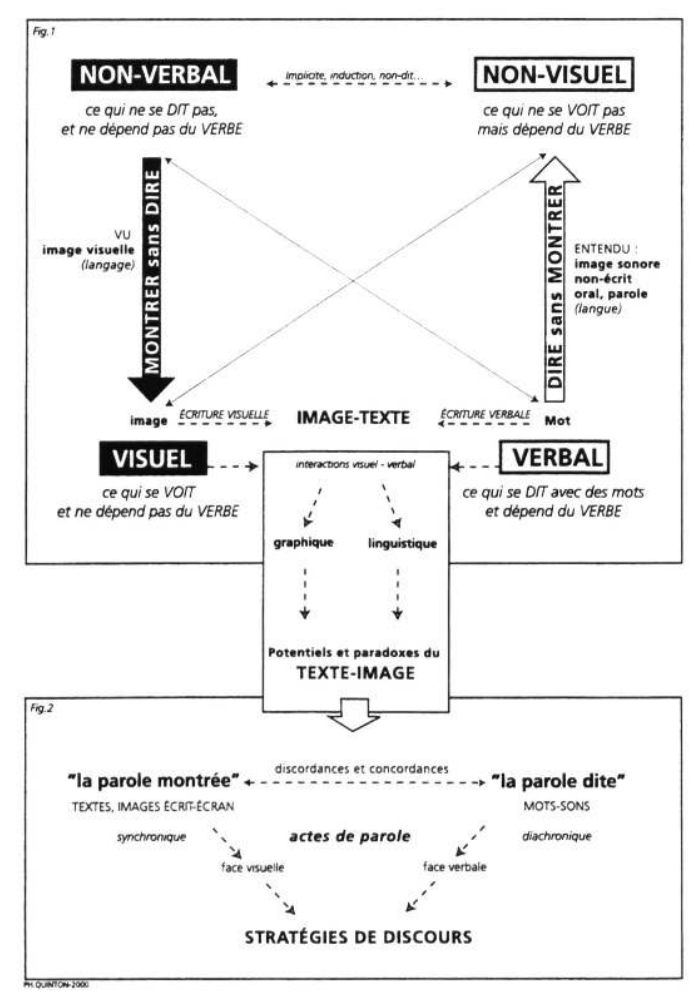




\section{Les potentiels non-verbaux du design}

Les signes non-verbaux permettent, en apparence, de se soustraire à la raison dominante de la langue, d'échapper à la supposée linéarité de l'écrit pour accéder à la fameuse tabulanté visuelle. Les traditions de l'imprimé ${ }^{10}$ se sont construites sur la matérialité visuelle du texte, elles sont aujourd'hui dynamisées dans la communication numérique avec de nouvelles procédures d'inscription qui appellent d'autres stratégies de «lecture ». Les écrans numériques brouillent les frontières entre verbal et visuel, l'image fixe ne l'est plus et "l'hypermédia " joue avec les synergies de ses faces graphiques, linguistiques, ou sonores pour créer des paroles d'écran bien spécifiques. Ainsi, le design des "écrits d'écran ${ }^{11}$ oblige à reconsidérer les hiérarchies et les habitudes acquises dans la communication graphique. Bien au-delà de l'esthétique des interfaces mises en place, une démarche de communication graphique est nécessairement structurée, organisée, elle n'est pas qu'apparence formelle, car nonverbal ne veut pas dire non-rationnel.

10 Pour les organisations, les atouts stratégiques du design sont désormais reconnus et attestés ${ }^{12}$. Outre ses fonctions d'identification, la dimension visuelle permet de positionner les modalités de l'interaction ou de la médiation avant celles de la parole ou de la lecture; d'esquiver le contrôle de la langue, bien que dans le moulin à paroles des médias le verbal veille toujours au grain. Plus l'image résiste, plus on en parle.

11 Les designs mettent en forme des concepts, ils montrent sans avoir besoin de dire, avec une capacité à initier et accompagner le changement ${ }^{13}$ dans les organisations. La démarche créative et conceptuelle du designer est un processus raisonné qui courtcircuite le verbal pour entrer de suite, et d'une manière globale, dans la forme et la substance iconique, la matière et la signification. Comme visualisation précoce des projets en amont des mises en production, la démarche design permet de formuler autrement les questions stratégiques que l'organisation doit traiter. Elle est en ce sens un facteur de décision pour un entrepreneur qui doit montrer la voie, voir à l'avance.

12 Par ailleurs, les productions formelles de l'organisation sont des vecteurs d'identification immédiate, mais aussi des prétextes aux discours sur l'organisation elle-même, comme par exemple pour Apple et ses nouvelles machines (iMac, iBook...) dont le design novateur fait beaucoup parler dans les médias à propos des projets et des valeurs de la firme. L'objet devient ainsi le support d'une production verbale sur un autre sujet.

\section{Les stratégies non-verbales d'identification}

13 Les processus d'identification sont indispensables à la vie d'un individu ou d'une organisation qui évoluent diversement dans des territoires physiques et symboliques, avec des impératifs de différenciation qui engagent le recours aux dimensions verbales et non-verbales pour assurer les fonctions sociales essentielles d'appartenance et de reconnaissance.

14 La reconnaissance est un processus qui permet de différencier une entité d'une autre. On demande ainsi aux systèmes d'identification visuelle d'assurer la différence et la permanence pour une organisation, le processus de reconnaissance se réalisant à 
travers des traits pertinents (des marques graphiques comme les logotypes) qui sont supposés symboliser une identité.

L'appartenance est un état caractérisant les relations d'un individu, d'un groupe ou d'une institution avec d'autres. Il s'agit de s'identifier à quelque chose en le manifestant visuellement (ce qui est le champ d'action préféré des marques commerciales).

\section{Signes d'existence et de reconnaissance}

16 La communication des organisations génère de nombreuses paroles graphiques qui ont pour mission d'interagir avec des sujets ou même d'autres systèmes afin de réaliser aussi bien des objectifs commerciaux ou institutionnels que des projets individuels. Il est clair que les manifestations non-verbales ne sont pas indépendantes des jeux de pouvoir des acteurs. En effet, si la communication visuelle met apparemment le sujet parlant à l'écart, contrairement à l'oral, elle n'évacue pas pour autant les annexions implicites du message à des fins stratégiques individuelles ou collectives. Identifier une entreprise c'est aussi reconnaître l'action personnelle d'un entrepreneur qui y laisse de nombreuses marques de son passage, traces qu'un successeur veut en général effacer...

Dire que l'on existe consiste d'abord à le montrer. Ainsi, la création des signes graphiques peut parfois précéder celle de leur référent institutionnel, comme dans le cas d'une entreprise en cours de création pour laquelle l'entrepreneur souhaite avoir rapidement un logotype et une carte de visite (la carte ferait ainsi préexister le territoire); ou encore la production d'un logo qui confère une surface médiatique ou rend plus crédible une opération ponctuelle. Cela donne l'impression que l'existence ne vaut qu'à travers ses manifestations visuelles, comme pour cet entrepreneur qui s'identifie à son entreprise en disant: "je n'existe pas, je n'ai pas de logo... » La confusion entre image (la représentation mentale) et image graphique (la marque visuelle qui se veut représentative d'une identité), est d'ailleurs assez fréquente, en particulier lorsque l'on affirme que « le logo est l'image de l'entreprise ».

18 À travers un dispositif graphique (cartes de visites, vêtements, objets, badges, voitures, etc.), un sujet membre d'une organisation tend à montrer qui il est, à quelles valeurs il se réfère, à quelle classe il appartient, en précisant aussi la place d'où il parle et agit. Les systèmes graphiques servent les intérêts des acteurs qui les pilotent selon des stratégies souvent fort différentes de celles qui leur sont officiellement affectées, ce que peut attester l'examen minutieux des systèmes d'identification visuelle des organisations et de leur évolution dans le temps. Par exemple, les modifications successives d'un système d'identification visuelle peuvent montrer la concomitance des changements de logos avec ceux des directions (La Villette). Les acteurs influents de l'organisation cherchent à prendre et à affirmer leur place dans les messages, veulent marquer visuellement leur territoire, défendre leur position avec des balises visuelles qui jalonnent le temps d'un mandat ou l'espace d'autorité d'une position hiérarchique.

19 Les discours graphiques ont pour objectif de "faire reconnaître ", de construire des relations en "faisant passer " les messages d'une organisation, ils tendent de la sorte à pré-construire des situations d'échange par des "mises en places» visuelles qui préparent le terrain et servent même parfois à esquiver les problèmes (ce sont alors des leurres).

On voit aussi un "changement d'identité » (entendez "visuelle ») faire l'objet d'une campagne de publicité, comme si le changement de logo n'était qu'un prétexte pour 
investir l'espace public, au même titre qu'une lessive qui vante sa nouveauté à l'occasion d'un changement d'emballage...

21 Instrumentalisés dans les marquages et les pondérations des ambitions, les signes graphiques de reconnaissance servent de monnaie d'échange pour calmer les appétits de pouvoir, préparer et orienter des négociations. Quand un opposant est " construit » sur le papier, il ne s'oppose plus de la même manière... L'image permet « d'abaisser la garde » en faisant appel au registre sensitif. Une concession visuelle dans le marquage des territoires hiérarchiques (accepter qu'un service et son chef disposent en propre d'un logo) peut canaliser la quête d'autorité et de territoire d'acteurs trop expansifs.

Une communication graphique peut aussi aider à recadrer, à reformuler un problème pour résoudre des conflits $^{14}$. Ce qui ne peut s'énoncer verbalement peut l'être graphiquement, cela permet de dire les choses autrement pour mieux les faire passer (voir l'expression " un dessin vaut mieux qu'un long discours »).

23 Mais les faits graphiques générés par une organisation ne sont pas tous des messages pensés et contrôlés par la main du stratège. Il existe une communication «malgré soi » qui échappe à la maîtrise organisationnelle et qui contribue aussi à la construction de son image : un mur sale; des supports de communication improvisés en interne; des messages non coordonnés, etc. Une approche sémiographique ${ }^{15}$ peut fournir une lecture particulière de ces manifestations visuelles qui échappent au contrôle verbal.

\section{Logotype et consensus non-verbaux}

24 L'interprétation des potentiels de la communication graphique se fait majoritairement selon des critères d'efficacité et de rationalité dans un espace graphique, public ou privé, fortement investi par la raison marchande. Le champ visuel est quelque peu asséché par des impératifs communicationnels issus d'un consensus qui veut " ratisser large ", comme c'est le cas pour les logotypes qui sont conçus selon des combinatoires formelles très conventionnelles. Ces objets graphiques à forte valeur stratégique, sont soumis aux dérives lexicales d'un pseudo "langage " visuel qui entend évacuer toute polysémie gênante en grammaticalisant les formes et les couleurs dans des connotations figées. Le repli sur les solutions courantes, lisses, consensuelles et sans risques, renforce nécessairement les conceptions stéréotypées sur ce que doit être un logo et empêche ce système graphique stratégique de se libérer d'un graphisme universel très convenu. De plus, pour parer à toute interprétation erronée, les concepteurs des logotypes accompagnent ceux-ci de discours qui disent comment le commanditaire et le destinataire doivent voir ce qu'on leur montre, ce qui est pris comme vérité officielle et souvent relayé tel quel par la plupart des journalistes... Cette nécessité de verbalisation témoigne des difficultés culturelles des décideurs (et de la société en général) en matière de communication visuelle. Le potentiel créatif est retenu par leurs petites peurs face à ce qui dépasse quelque peu le " raisonnable ».

L'opposition verbal-visuel peut aussi apparaître dans le jeu entre la classe et l'un de ses membres ${ }^{16}$ comme le montrent par exemple les logos des IUT de France qui manifestent tous - grâce au sigle-nom commun - leur appartenance à la catégorie institutionnelle «IUT» (le verbal du sigle se rapporte à la classe "IUT»), tout en affirmant visuellement leurs spécificités avec leurs interprétations graphiques de cet intitulé fédérateur (le visuel assure la reconnaissance de l'établissement). 
26 Au-delà du logo, le non-verbal peut gérer une cohabitation très ordinaire entre appartenance et reconnaissance: comme pour cette boulangerie franchisée sous une enseigne répandue. L'agencement du magasin et l'approche commerciale manifestent une appartenance au réseau qui apporte en retour une référence et une image de qualité soutenue par une publicité nationale. Mais l'artisan boulanger veut être reconnu sous cette tutelle, défendre son ego de commerçant. Il exploite ainsi les quelques marges de manœuvre dont il dispose pour la personnalisation de sa boutique en gravant notamment ses initiales en lettres capitales (un Garamond à connotation royale) dans le marbre de la poignée de porte. La typo met ainsi en place une stratégie de réapropriation de territoire...

\section{Le statut numérique du non-verbal}

Dans le domaine informatique, on sait que les logiciels pré-construisent à leur manière la mise en forme de nos communications, si ce n'est notre manière de les concevoir, ce qui conditionne les objets qui en résultent. Nous prenons volontiers les réglages « par défaut" pour la norme acquise sans essayer de les reconfigurer, ce qui installe l'évidence de ces normes dans les implicites de nos productions écrites et visuelles, les contraintes non-verbales étant facilement déléguées aux technologies et à leurs procédures préconstruites.

Par exemple, les consignes de mise en forme fournies pour la présente communication ne disent rien sur les données de base (visuelles) de la composition: le format de la page, l'empagement du texte courant (les marges), le corps, etc. Il apparait que ces données (implicites) correspondent aux réglages «par défaut » du logiciel cité, et bien qu'essentielles à l'aspect du document, semblent donc laissées à la main des concepteurs de ce logiciel. Par contre les indications concernant la rédaction (le verbal) sont plus précises. En quelque sorte, les données non-verbales seraient gérées par défaut, alors que le verbal se verrait davantage prescrit. Les chercheurs en communication auraient-ils des difficultés à penser et gérer l'image aussi bien que le texte?

\section{Laisser la parole au non-verbal}

Dans les communications d'aujourd'hui, les messages en image-texte établissent des relations paradoxales entre visuel et verbal, ce qui ouvre des potentiels spécifiques dans les stratégies d'identification. Mais si notre société se veut effectivement de "l'image ", c'est une image asservie et appauvrie. Il conviendrait sans doute de laisser au non-verbal l'espace graphique qui est le sien en le soustrayant de la tutelle verbale et en cessant de l'instrumentaliser à outrance. En matière d'identification, la différence, la reconnaissance et l'appartenance s'établissent sous l'égide d'un consensus graphique universel qui nivelle les expressions. Une démarche d'autonomisation des potentialités visuelles ne peut que s'inscrire dans la réflexion sur les nouvelles modalités d'écriture et de lecture qui se mène aujourd'hui et de leurs implications stratégiques dans l'interconnexion générale qui consacre la domination du vu sur le lu.

Les potentialités non-verbales ne jouissent pas du même crédit rationnel et ne sont pas gérées aussi bien que les approches verbales jugées plus "contrôlables ». Ce sous- 
développement notable de la maîtrise iconique est aggravé par les facilités combinatoires des écrans qui laissent chacun devant ses illusions de «co-créateur d'images » dans la manipulation « créative » de pixels. Les designs des représentations et des inscriptions exigent avant tout la maitrise du dessin (l'exercice du regard), ainsi que la pertinence et l'éthique propres au dessein (le projet) qui pilote normalement toute production visuelle originale.

Il nous reste d'une part, à œuvrer pour que la dimension visuelle occupe la place qu'elle mérite dans les enseignements et les pratiques de communication ${ }^{17}$, et d'autre part à inciter les organisations à se montrer et se dire pertinemment, en valorisant les potentiels et la raison sensible d'une dimension non-verbale essentielle à leur existence.

\section{NOTES}

1. À la fois matériel et symbolique, il permet d'assembler des données hétérogènes pour les communiquer. Voir : Le dispositif, entre usages et concept, Hermès $n^{\circ} 25$, CNRS éditions 1999.

2. Groupe Mu, Traité du signe visuel. Seuil, 1992, p. 146

3. Il est en effet très rare de voir une telle analyse formulée uniquement avec des images, bien que cela soit parfaitement possible (voir les pratiques des plasticiens).

4. Entendu ici hors de toute considération psychologique, l'image est elle aussi indicible...

5. L'action de montrer implique un projet et une décision de la part d'un émetteur, bien que tous les faits graphiques ne soient pas intentionnels, ce qui ne se voit pas forcément en réception.

6. Éliséo Véron, Quand dire c'est faire, renonciation dans le discours de la presse écrite. Sémiotique II, IREP 1986

7. Jean-Pierre Meunier, "Connaître par l'image », in Images et cognition, UCL 1998

8. Bernard Darras « L'image, une vue de l'esprit », in dans Images et cognition. UCL 1998

9. La dimension cachée, Seuil 1971.

10. Relatées notamment dans L'aventure des écritures, la page. Bibliothèque Nationale France, 1999.

11. Yves Jeanneret, Emmanuel Souchier, Pour une poétique de "l'écrit » d'écran. Xoana n 6, p. 97 à 100, Éd. J.M. Place 1999.

12. P. Rasquinet, M. Brun, L'identité visuelle de l'entreprise. Éd. d'organisation, 1996.

13. Sur ce sujet, voir Philippe Quinton, Design graphique et changement. L'Harmattan 1996.

14. En référence aux travaux de Paul Watzlawick, évidemment.

15. Qui consiste à faire un relevé et une description méthodique des faits visuels relatifs à une organisation.

16. Selon les termes de Paul Watzlawick, Changements et Logique de la communication. Seuil 1975/1972

17. Voir Peut-on apprendre à voir ? sous la direction de Laurent Gervereau, L'image/ENSBA 1999. 


\section{AUTEUR}

\section{PHILIPPE QUINTON}

Université Pierre Mendès France, Grenoble II - Centre d'études de l'écriture, Paris VII (EP 2044) 